\title{
DYNAMIC RANDOM EVOLUTIONS ON INCREASING TIME INTERVALS
}

UDC 519.21

\author{
V. S. KOROLIUK
}

\begin{abstract}
We consider the following three main limit theorems for random evolutions: the averaging scheme, diffusion approximation, and asymptotic behavior of large deviations.

We study the asymptotic behavior of random evolutions in increasing time intervals in the scheme of series with a small parameter by using solutions of the singular perturbation problem for reducible-invertible operators.
\end{abstract}

\section{INTRODUCTION}

We study the asymptotic behavior of random evolutions in increasing time intervals by using solutions of the singular perturbation problem for reducible-invertible operators; see [1. Chapter 5]. To justify the existence of a limit, we use the martingale characterization of Markov processes and conditions of the relative compactness; see [3].

The asymptotic behavior of random evolutions is studied in the scheme of series with a small parameter, $\varepsilon \rightarrow 0, \varepsilon>0$; see [1, Chapter 3]. The main object of the asymptotic analysis for random evolutions is the generating operator (generator) of the corresponding Markov process; see [1, 3].

The following three types of limit theorems are known in the theory of stochastic processes: the averaging scheme or law of large numbers, diffusion approximation or central limit theorem, and asymptotic behavior of large deviations or exponential estimate of small probabilities. Each type of limit theorem corresponds to its own normalization condition given in terms of the parameter $\varepsilon$.

It is unfeasible for practical purposes to list here all the literature on limit theorems for stochastic processes in general and for random evolutions in particular. The best solution for the reader is to look at the monographs mentioned at the end of this paper and at the references therein.

\section{Dynamic RANDOM EVOlutions}

Markov random evolutions are defined by a solution of a dynamic system of differential equations

$$
d u(t) / d t=C(u(t) ; x(t)), \quad u(0)=u_{0} \in \mathbb{R}^{d},
$$

in the Euclidean space $\mathbb{R}^{d}, d \geq 1$. The speed of the evolution $C(u ; x), u \in \mathbb{R}^{d}, x \in E$, depends on states $x \in E$ of a regular homogeneous Markov jump process $x(t), t \geq 0$,

2000 Mathematics Subject Classification. Primary 60J55, 60B10, 60F17, 60K10; Secondary 60G46, $60 \mathrm{G} 60$.

Key words and phrases. Dynamic random evolution, averaging, diffusion approximation, large deviations, exponential nonlinear operator. 
corresponding to the generator

$$
Q \varphi(x)=q(x) \int_{E} P(x, d y)[\varphi(y)-\varphi(x)], \quad \varphi(x) \in \mathcal{B}_{E}
$$

The generator acts at real valued test functions with the norm $|\varphi|:=\sup _{x \in E}|\varphi(x)|$; see [6, 7].

It is known (see, for example, 6, 7]) that the function $q(x)$ in (2) defines the intensity of the sojourn time of $\theta_{x}$ in the states $x \in E$ :

$$
\mathrm{P}\left\{\theta_{x} \geq t\right\}=e^{-q(x) t}, \quad t \geq 0 .
$$

The stochastic kernel $P(x, d y), x \in E, d y \in \mathcal{E}$, defines the transition probabilities for the embedded Markov chain $x_{n}=x\left(\tau_{n}\right), n \geq 0$ :

$$
\mathrm{P}\left\{x_{n+1} \in B \mid x_{n}=x\right\}=P(x, B), \quad x \in E, B \in \mathcal{E} .
$$

The renewal moments (of jumps) $\tau_{n}, n \geq 0$, are defined by

$$
\tau_{n+1}=\tau_{n}+\theta_{n+1}, \quad n \geq 0 .
$$

Therefore

$$
\mathrm{P}\left\{\theta_{n+1} \geq t \mid x_{n}=x\right\}=\mathrm{P}\left\{\theta_{x} \geq t\right\}=e^{-q(x) t} .
$$

The accompanying evolutionary systems

$$
d u_{x}(t) / d t=C\left(u_{x}(t) ; x\right), \quad x \in E,
$$

are characterized by the generators

$$
\mathbf{C}(x) \varphi(u)=C(u ; x) \varphi^{\prime}(u), \quad \varphi(u) \in C^{1}\left(\mathbb{R}^{d}\right),
$$

of the semigroups

$$
\mathbf{C}_{t}(x) \varphi(u):=\varphi\left(u_{x}(t)\right), \quad u_{x}(0)=u
$$

see [2, Chapter 4].

The Markov random evolution (1) -(2) is characterized by the generator [2, $\S 4.2]$ of the two component Markov process $u(t), x(t), t \geq 0$ :

$$
\mathbf{L} \varphi(u, x)=Q \varphi(\cdot, x)+\mathbf{C}(x) \varphi(u, \cdot) .
$$

\section{RANDOM EVOLUTIONS IN THE SCHEME OF SERIES}

We study the following three types of the scheme of series with a small positive parameter $\varepsilon \rightarrow 0, \varepsilon>0$ :

(1) averaging scheme,

(2) diffusion approximation, and

(3) large deviations with an asymptotically small diffusion.

The following is the main assumption we impose on the random evolutions in the scheme of series.

Main assumption. The Markov switching process $x(t), t \geq 0$, in a standard phase space $(E, \mathcal{E})$ is uniformly ergodic with a unique stationary distribution $\pi(B), B \in \mathcal{E}$.

The stationary distribution of the embedded Markov chain $x_{n}, n \geq 0$, is defined by

$$
\begin{gathered}
\pi(d x) q(x)=q \rho(d x), \quad q=\int_{E} \pi(d x) q(x), \\
\rho(B)=\int_{E} \rho(d x) P(x, d y), \quad \rho(E)=1 .
\end{gathered}
$$


3.1. Averaging. Dynamic random evolutions in an averaging scheme are described by a solution of a dynamic system,

$$
d u^{\varepsilon}(t) / d t=C\left(u^{\varepsilon}(t) ; x(t / \varepsilon)\right), \quad u^{\varepsilon}(0)=u_{0} \in \mathbb{R}^{d}
$$

with Markov switchings $x_{t}^{\varepsilon}:=x(t / \varepsilon)$ defined in increasing time intervals. The normalization in this case is given by $T_{\varepsilon}=t / \varepsilon, \varepsilon \rightarrow 0$.

The two component Markov process

$$
u^{\varepsilon}(t), x_{t}^{\varepsilon}=x(t / \varepsilon), \quad t \geq 0, \varepsilon \rightarrow 0,
$$

corresponds to the generator

$$
L^{\varepsilon} \varphi(u, x)=\left[\varepsilon^{-1} Q+\mathbf{C}(x)\right] \varphi(u, x) .
$$

The asymptotic analysis of the random evolution (3) characterized by generator (4) is based on the solution of the problem of the singular perturbation for the reducibleinvertible operator $Q$; see [1, Chapter 5].

The uniform ergodicity of the Markov switching process $x(t), t \geq 0$, implies that the operator $Q$ is reducible-invertible. In other words, the uniform ergodicity implies that a bounded potential $R_{0}$ exists and is defined by the equation

$$
Q R_{0}=R_{0} Q=\Pi-I, \quad \Pi \varphi(x):=\int_{E} \pi(d x) \varphi(x)
$$

(see [1, Chapter 5]).

Thus the Poisson equation

$$
Q \varphi(x)=\psi(x), \quad \Pi \psi(x)=0,
$$

has a unique solution that can be determined from the equalities

$$
\varphi(x)=-R_{0} \psi(x), \quad \Pi \varphi(x)=0 .
$$

The asymptotic representation of the generator (44) of the random evolution (3) is defined at the perturbed test function

$$
\varphi^{\varepsilon}(u, x)=\varphi(u)+\varepsilon \varphi_{1}(u, x) .
$$

Lemma 3.1. The asymptotic representation

$$
\mathbf{L}^{\varepsilon} \varphi^{\varepsilon}(u, x)=\widehat{C} \varphi(u)+\delta_{l}^{\varepsilon}(x) \varphi(u)
$$

holds with the negligible term

$$
\sup _{x \in E}\left|\delta_{l}^{\varepsilon}(x) \varphi(u)\right| \rightarrow 0, \quad \varepsilon \rightarrow 0,
$$

where $\varphi(u) \in C^{2}\left(\mathbb{R}^{d}\right)$. The limit operator is given by the relations

$$
\widehat{C} \varphi(u)=\widehat{C}(u) \varphi^{\prime}(u), \quad \widehat{C}(u)=\int_{E} \pi(d x) C(u ; x) .
$$

The asymptotic representation (5) follows explicitly from Proposition 5.1 in [1, Chapter 5] with $Q_{1}=\mathbf{C}(x)$.

The asymptotic representation (5) is used to prove the weak convergence

$$
u^{\varepsilon}(t) \Rightarrow \widehat{u}(t), \quad \varepsilon \rightarrow 0 .
$$

The limit evolution corresponds to a solution of the averaged evolution equation

$$
d \widehat{u}(t) / d t=\widehat{C}(\widehat{u}(t)), \quad \widehat{u}(0)=\widehat{u}_{0} \in \mathbb{R}^{d},
$$

(see [1, Chapters 3, 5, 6]). 
Remark 3.1. The weak convergence (6) is a variant of the "averaging principle" for stochastic systems that has a long history starting with H. Poincaré, M. M. Bogolyubov, I. I. Gikhman, etc.

3.2. Diffusion approximation. Dynamic random evolutions in the scheme of diffusion approximation correspond to a solution of the dynamical system

$$
d u^{\varepsilon}(t) / d t=C^{\varepsilon}\left(u^{\varepsilon}(t) ; x\left(t / \varepsilon^{2}\right)\right), \quad u^{\varepsilon}(0)=u_{0} \in \mathbb{R}^{d},
$$

with Markov switchings $x_{t}^{\varepsilon}:=x\left(t / \varepsilon^{2}\right)$. Increasing time intervals for this scheme we get other normalizations, namely $T_{\varepsilon}=t / \varepsilon^{2}, \varepsilon \rightarrow 0$.

The speed of evolution still depends on a small parameter of the scheme of series, namely

$$
C^{\varepsilon}(u ; x)=C(u ; x)+\varepsilon^{-1} C_{0}(u ; x), \quad u \in \mathbb{R}^{d}, x \in E .
$$

The first component $C(u ; x)$ generates the averaged evolution, while the second component, $C_{0}(u ; x)$, generates diffusion fluctuations under an additional balance condition:

$$
\Pi C_{0}(u ; x)=\int_{E} \pi(d x) C_{0}(u ; x) \equiv 0 .
$$

The Markov random evolution (77)-(8) is characterized by the generator

$$
\mathbf{L}^{\varepsilon} \varphi(u, x)=\left[\varepsilon^{-2} Q+\varepsilon^{-1} \mathbf{C}_{0}(x)+\mathbf{C}(x)\right] \varphi(u, x)
$$

of the two component Markov process $u^{\varepsilon}(t), x_{t}^{\varepsilon}:=x\left(t / \varepsilon^{2}\right), t \geq 0$.

Here

$$
\mathbf{C}_{k}(x) \varphi(u):=C_{k}(u ; x) \varphi^{\prime}(u), \quad k=0,1 .
$$

The asymptotic analysis of the random evolution (7)-(8) under the balance condition (9) is based on a solution of the singular perturbation problem for the reducibleinvertible operators $Q$ corresponding to the uniformly ergodic Markov switching process; see [1, Chapter 5].

The asymptotic representation of the generator (10)-(11) is considered at test functions

$$
\varphi^{\varepsilon}(u, x)=\varphi(u)+\varepsilon \varphi_{1}(u, x)+\varepsilon^{2} \varphi_{2}(u, x) .
$$

Lemma 3.2. Let the balance condition (9) hold. Then

$$
\mathbf{L}^{\varepsilon} \varphi^{\varepsilon}(u, x)=\widehat{\mathbf{L}} \varphi(u)+\delta_{l}^{\varepsilon}(x) \varphi(u)
$$

where the negligible term is

$$
\sup _{x \in E}\left|\delta_{l}^{\varepsilon}(x) \varphi(u)\right| \rightarrow 0, \quad \varepsilon \rightarrow 0,
$$

for $\varphi(u) \in C^{3}\left(\mathbb{R}^{d}\right)$. The limit operator $\widehat{\mathbf{L}}$ is evaluated from

$$
\widehat{\mathbf{L}}=\Pi \mathbf{C}(x) \Pi+\Pi \mathbf{C}_{0}(x) R_{0} \mathbf{C}_{0}(x) \Pi .
$$

The asymptotic representation (12)-(13) follows explicitly from Proposition 5.2 of 1 , Chapter 5] with $Q_{1}=\mathbf{C}_{0}(x)$ and $Q_{2}=\mathbf{C}(x)$.

Conclusion 3.1. The asymptotic representation (12)-(13) is used to prove the weak convergence

$$
u^{\varepsilon}(t) \Rightarrow \zeta(t), \quad \varepsilon \rightarrow 0
$$

(see [1, Chapter 6]). 
The limit diffusion process $\zeta(t), t \geq 0$, corresponds to the generator

$$
\begin{gathered}
\widehat{L} \varphi(u)=b(u) \varphi^{\prime}(u)+\frac{1}{2} B(u) \varphi^{\prime \prime}(u), \\
b(u)=\widehat{C}(u)+\widehat{C}_{0}(u), \quad \widehat{C}_{0}(u)=\Pi C_{0}(u ; x) R_{0} C_{0}^{\prime}(u ; x) \Pi, \\
B(u)=2 \Pi C_{0}(u ; x) R_{0} C_{0}(u ; x) \mathbf{1} .
\end{gathered}
$$

Here

$$
C_{0}^{\prime}(u ; x):=\frac{\partial C_{0}(u ; x)}{\partial u} .
$$

Remark 3.2. The weak convergence (14) is a variant of the diffusion approximation of fluctuations of stochastic systems studied for a long time starting with R. Z. Khas'minskil.

Remark 3.3. The local balance condition (9) can sometimes be replaced by another condition. For example, this can be the condition of "balance with equilibrium" (see [1, $\S \S 3.5,5.5])$. Note that the procedure of evaluating the limit operator becomes much more complicated in this case. The meaning of formula (13) is also changed, as explained in 1, $\S 5.5 .2]$.

Remark 3.4. The procedures for the averaging scheme and diffusion approximation for dynamic random evolutions with a Markov switching process $x(t), t \geq 0$, are also described in [1].

3.3. Asymptotically small diffusion. The dynamic random evolution in the scheme of asymptotically negligible diffusion corresponds to a solution of the following evolution equation:

$$
d u^{\varepsilon}(t) / d t=C\left(u^{\varepsilon}(t) ; x\left(t / \varepsilon^{3}\right)\right)+\varepsilon^{-1} C_{0}\left(x\left(t / \varepsilon^{3}\right)\right) .
$$

The perturbed speed

$$
C^{\varepsilon}(u ; x)=C(u ; x)+\varepsilon^{-1} C_{0}(x), \quad u \in \mathbb{R}^{d}, x \in E,
$$

satisfies the balance condition

$$
\Pi C_{0}(x)=\int_{E} \pi(d x) C_{0}(x)=0 .
$$

The Markov random evolution (15)-(17) is characterized by the generator

$$
\mathbf{L}^{\varepsilon} \varphi(u, x)=\left[\varepsilon^{-3} Q+\varepsilon^{-1} \mathbf{C}_{0}(x)+\mathbf{C}(x)\right] \varphi(u ; x)
$$

of the two component Markov process $u^{\varepsilon}(t), x_{t}^{\varepsilon}:=x\left(t / \varepsilon^{3}\right), t \geq 0$.

By definition,

$$
\mathbf{C}_{0}(x) \varphi(u)=C_{0}(x) \varphi^{\prime}(u), \quad \mathbf{C}(x) \varphi(u)=C(u ; x) \varphi^{\prime}(u) .
$$

The asymptotic analysis of the random evolution (15)-(17) is based on the same idea as that used in Section 3.2, namely, the generator (18)-(19) is considered at the perturbed test functions

$$
\varphi^{\varepsilon}(u, x)=\varphi(u)+\varepsilon \varphi_{1}(u, x)+\varepsilon^{3} \varphi_{2}(u, x) .
$$

Lemma 3.3. Let the balance condition (17) hold. Then the generator (18)-(19) admits the asymptotic representation

$$
\mathbf{L}^{\varepsilon} \varphi^{\varepsilon}(u, x)=\widehat{\mathbf{L}}^{\varepsilon} \varphi(u)+\delta_{l}^{\varepsilon}(x) \varphi(u)
$$

with the negligible term

$$
\sup _{x \in E}\left|\delta_{l}^{\varepsilon}(x) \varphi(u)\right| \rightarrow 0, \quad \varepsilon \rightarrow 0, \varphi(u) \in C^{3}\left(\mathbb{R}^{d}\right) .
$$


The averaged operator $\widehat{\mathbf{L}}^{\varepsilon}$ is evaluated from

$$
\widehat{L}^{\varepsilon}=\Pi \mathbf{C}(x) \Pi+\varepsilon \Pi \mathbf{C}_{0}(x) R_{0} \mathbf{C}_{0}(x) \Pi .
$$

The result of Lemma 3.3 becomes obvious if one rewrites the generator (18) in the form

$$
\mathbf{L}^{\varepsilon}=\varepsilon^{-3} Q+\varepsilon^{-2} \mathbf{C}_{0}^{\varepsilon}(x)+\mathbf{C}(x), \quad \mathbf{C}_{0}^{\varepsilon}(x):=\varepsilon \mathbf{C}_{0}(x),
$$

and applies Lemma 3.2.

Note that the asymptotic representation (20) means that

$$
u^{\varepsilon}(t) \simeq \zeta^{\varepsilon}(t), \quad \varepsilon \rightarrow 0
$$

where the diffusion process $\zeta^{\varepsilon}(t)$ is a solution of the following stochastic differential equation:

$$
d \zeta^{\varepsilon}(t)=\widehat{C}\left(\zeta^{\varepsilon}(t)\right) d t+\sqrt{\varepsilon} \sigma d w(t) .
$$

The matrix of variations is given by

$$
B=\sigma^{*} \sigma, \quad B=2 \int_{E} \pi(d x) C_{0}(x) R_{0} C_{0}(x) .
$$

We use the asymptotic representation (21)-(23) to solve the problem of large deviations for dynamic random evolutions (see Section 4 below).

\section{LARGE DEVIATIONS FOR DYNAMIC RANDOM EVOLUTIONS IN THE SCHEME OF ASYMPTOTICALLY SMALL DIFFUSION}

The large deviations or asymptotic behavior of small probabilities for random evolutions is studied by using the method of the asymptotic analysis of the exponential (nonlinear) generator of large deviations developed in [4.

The exponential generator $H^{\varepsilon}$ of large deviations for a Markov process corresponding to the generator $L^{\varepsilon}$ in the scheme of series is defined by

$$
H^{\varepsilon} \varphi(u)=e^{-\varphi(u) / \varepsilon} \varepsilon L^{\varepsilon} e^{\varphi(u) / \varepsilon}
$$

(see [4, Part I]). The theory of large deviations for stochastic processes is based on the characterization of a Markov process by the exponential martingale

(see [4, Part I]).

$$
\exp \left\{\varphi(u(t))-\varphi(u(0))-\int_{0}^{t} H \varphi(u(s)) d s\right\}=\mu_{t}
$$

The exponential generator $H$ in the latter relation is given by

$$
H \varphi(u)=e^{-\varphi(u)} L e^{\varphi(u)},
$$

where $L$ is the generator of the Markov process $u(t), t \geq 0$.

The large deviations problem for Markov processes can be understood as a limit theorem in the scheme of series with a small parameter $\varepsilon \rightarrow 0, \varepsilon>0$, namely

$$
H^{\varepsilon} \varphi^{\varepsilon} \rightarrow H \varphi, \quad \varphi^{\varepsilon} \rightarrow \varphi, \quad \varepsilon \rightarrow 0
$$

Example 4.1. Let the asymptotically small diffusion $\zeta^{\varepsilon}(t)=\sqrt{\varepsilon} \sigma w(t), t \geq 0$, be defined by the generator

$$
L^{\varepsilon} \varphi(u)=\varepsilon \frac{1}{2} \sigma^{2} \varphi^{\prime \prime}(u) .
$$

Then the exponential generator $H^{\varepsilon}$ of the asymptotically small diffusion (26) is easy to derive from equality (24), namely

$$
H^{\varepsilon} \varphi(u)=\frac{1}{2} \sigma^{2}\left[\varphi^{\prime}(u)\right]^{2}+\varepsilon \sigma^{2} \varphi^{\prime \prime}(u) .
$$


This implies that the limit exponential generator of the asymptotically small diffusion (26) is given by

$$
H \varphi(u)=\frac{1}{2} \sigma^{2}\left[\varphi^{\prime}(u)\right]^{2}
$$

Remark 4.1. Without loss of generality, one may evaluate the limit exponential generator only in the one-dimensional Euclidean space $R$, that is, in the real line.

It is clear that the exponential generator (27) can be written in the space $R^{d}, d>1$, as

$$
H \varphi(u)=\frac{1}{2} \sum_{k, r=1}^{d} \sigma_{k r} \varphi_{k}^{\prime} \varphi_{r}^{\prime}, \quad \varphi_{k}^{\prime}:=\partial \varphi(u) / \partial u_{k}, \quad 1 \leq k \leq d .
$$

Here $\sigma^{2}=\left[\sigma_{k r} ; 1 \leq k, r \leq d\right]$ is the covariance matrix of the Brownian motion.

Dynamic random evolutions in the scheme of an asymptotically small diffusion are determined by a solution of the evolution equation (15) with an additional balance condition (17).

The random evolution (15) is characterized by the generator (18)-(19).

Theorem 4.1. Let the balance condition (17) hold. If the main assumption is satisfied, that is, if the Markov switching process $x(t), t \geq 0$, is uniformly ergodic, then the exponential generators converge, namely

$$
H^{\varepsilon} \varphi^{\varepsilon}(u, x) \rightarrow H \varphi(u), \quad \varphi^{\varepsilon}(u, x) \rightarrow \varphi(u), \quad \varepsilon \rightarrow 0,
$$

for perturbed test functions

$$
\varphi^{\varepsilon}(u, x)=\varphi(u)+\varepsilon \ln \left[1+\varepsilon \varphi_{1}(u, x)+\varepsilon^{2} \varphi_{2}(u, x)\right] .
$$

The limit exponential generator is given by

$$
\begin{gathered}
H \varphi(u)=\frac{1}{2} \sigma^{2}\left[\varphi^{\prime}(u)\right]^{2}+\widehat{C}(u) \varphi^{\prime}(u), \\
\sigma^{2}=2 \int_{E} \pi(d x) C_{0}(x) R_{0} C_{0}(x), \quad \widehat{C}(u)=\int_{E} \pi(d x) C(u ; x) .
\end{gathered}
$$

The proof of Theorem 4.1 is based on the asymptotic representation of exponential generators. This auxiliary result is stated below.

Lemma 4.1. The following asymptotic representation holds:

$$
H^{\varepsilon} \varphi^{\varepsilon}(u, x)=\varepsilon^{-1}\left[Q \varphi_{1}+\mathbf{C}_{0}(x) \varphi\right]+\left[Q \varphi_{2}-\varphi_{1} Q \varphi_{1}+\mathbf{C}(x) \varphi\right]+\delta_{h}^{\varepsilon}(x) \varphi(u),
$$

where the negligible term is such that

$$
\sup _{x \in E}\left|\delta_{h}^{\varepsilon}(x) \varphi(u)\right| \rightarrow 0, \quad \varepsilon \rightarrow 0, \quad \varphi(u) \in C^{3}(R) .
$$

Proof of Lemma 4.1. The proof follows from the asymptotic representations of the three operators defining the generator (18).

We have

$$
\begin{aligned}
H_{Q}^{\varepsilon} \varphi^{\varepsilon}(u, x) & =e^{-\varphi / \varepsilon}\left[1+\varepsilon \varphi_{1}+\varepsilon^{2} \varphi_{2}\right]^{-1} \varepsilon^{-2} Q\left[1+\varepsilon \varphi_{1}+\varepsilon^{2} \varphi_{2}\right] e^{\varphi / \varepsilon} \\
& =e^{-\varphi / \varepsilon}\left[1-\varepsilon \varphi_{1}-\varepsilon^{2} \varphi_{2}\right] \varepsilon^{-2} Q\left[1+\varepsilon \varphi_{1}+\varepsilon^{2} \varphi_{2}\right] e^{\varphi / \varepsilon}+\delta_{q}^{\varepsilon}(x) \varphi \\
& =\varepsilon^{-1} Q \varphi_{1}+Q \varphi_{2}-\varphi_{1} Q \varphi_{1}+\delta_{q}^{\varepsilon}(x) \varphi .
\end{aligned}
$$

Similarly we obtain the asymptotic representations

$$
\begin{aligned}
H_{0}^{\varepsilon} \varphi^{\varepsilon}(u, x) & =\varphi^{-\varphi / \varepsilon}\left[1+\varepsilon \varphi_{1}+\varepsilon^{2} \varphi_{2}\right]^{-1} \mathbf{C}_{0}(x)\left[1+\varepsilon \varphi_{1}+\varepsilon^{2} \varphi_{2}\right] e^{\varphi / \varepsilon} \\
& =\varepsilon^{-1} \mathbf{C}_{0}(x) \varphi+\delta_{0}^{\varepsilon}(x) \varphi
\end{aligned}
$$


and

$$
\begin{aligned}
H_{c}^{\varepsilon} \varphi^{\varepsilon}(u, x) & =e^{-\varphi / \varepsilon}\left[1+\varepsilon \varphi_{1}+\varepsilon^{2} \varphi_{2}\right]^{-1} \varepsilon \mathbf{C}(x)\left[1+\varepsilon \varphi_{1}+\varepsilon^{2} \varphi_{2}\right] e^{\varphi / \varepsilon} \\
& =\mathbf{C}(x) \varphi+\delta_{c}^{\varepsilon}(x) \varphi .
\end{aligned}
$$

Combining the results above we prove (29).

To complete the proof of Theorem 4.1 we use solutions of the singular perturbation problems

$$
\begin{gathered}
Q \varphi_{1}+\mathbf{C}_{0}(x) \varphi(u)=0 \\
Q \varphi_{2}-\varphi_{1} Q \varphi_{1}+\mathbf{C}(x) \varphi(u)=\widehat{\mathbf{L}} \varphi(u)
\end{gathered}
$$

(see [1, Chapter 5]).

The existence of a solution of equation (30) follows from balance condition (17). Therefore

$$
\varphi_{1}(u, x)=-R_{0} \mathbf{C}_{0}(x) \varphi(u)=-R_{0} C_{0}(x) \varphi^{\prime}(u) .
$$

Considering (30) we transform equation (31) as follows:

$$
Q \varphi_{2}+C_{0}(x) R_{0} C_{0}(x)\left[\varphi^{\prime}(u)\right]^{2}+C(u ; x) \varphi^{\prime}(u)=\widehat{\mathbf{L}} \varphi(u) .
$$

The limit operator $\widehat{\mathbf{L}}$ is determined from the solvability condition for equation (33). This, in particular, means that formula (28) holds.

Remark 4.2. In general, there are four steps in the solution of the large deviations problem for Markov processes as stated in [4, Chapter 2], namely:

(1) convergence of exponential generators,

(2) exponential density of Markov processes,

(3) comparison principle for the limit operator,

(4) construction of the variation representation for the action functional.

Since steps 2-4 are already done for the limit operator of asymptotically small diffusion (27) and even for a more general case of exponential generator (28) (see [4, 5]), we briefly present below only the first step of the proof, namely the convergence of exponential generators (25).

Remark 4.3. A similar large deviations problem is considered in the monograph [4, §11.3] for the case of two (large) parameters of the scheme of series, namely for $n \rightarrow \infty$ and $\beta_{n} \rightarrow \infty$ as $n \rightarrow \infty$. Passing to the small parameter of series $\varepsilon=1 / n=1 / \beta_{n}$, we obtain an analogous normalization for the generator

$$
L^{\varepsilon} \varphi(u, x)=\left[\varepsilon^{-3} Q+\varepsilon^{-1} C_{0}(x)\right] \varphi(u, x)
$$

of the two component Markov process. Nevertheless, the solution of the singular perturbation problem gives us the limit exponential operator that depends on the states of the Markov switching process.

Remark 4.4. A usual method to solve the classical large deviations problem for a diffusion process with an asymptotically small diffusion,

$$
d u^{\varepsilon}(t)=\widehat{C}\left(u^{\varepsilon}(t)\right) d t+\sqrt{\varepsilon} \sigma d w(t),
$$

is to use the exponential generator

$$
H^{\varepsilon} \varphi(u)=e^{-\varphi(u) / \varepsilon} \varepsilon L^{\varepsilon} e^{\varphi(u) / \varepsilon}
$$

and the generator of the process (22),

$$
L^{\varepsilon} \varphi(u)=\widehat{C}(u) \varphi^{\prime}(u)+\varepsilon \frac{1}{2} \sigma^{2} \varphi^{\prime \prime}(u) .
$$


Then the solution is given by

where

$$
\begin{gathered}
H_{c}^{\varepsilon} \varphi(u):=e^{-\varphi / \varepsilon} \varepsilon \widehat{\mathbf{C}} e^{\varphi / \varepsilon}=\widehat{C} \varphi(u)=\widehat{C}(u) \varphi^{\prime}(u), \\
H_{\sigma}^{\varepsilon} \varphi(u):=e^{-\varphi / \varepsilon} \varepsilon^{2} \mathbf{B} e^{\varphi / \varepsilon}=\frac{1}{2} \sigma^{2}\left[\varphi^{\prime}(u)\right]^{2}+\varepsilon \frac{1}{2} \sigma^{2} \varphi^{\prime \prime}(u),
\end{gathered}
$$

$$
\mathbf{B} \varphi(u):=\frac{1}{2} \sigma^{2} \varphi^{\prime \prime}(u) .
$$

Therefore we obtain the convergence of the exponential generators,

$$
H^{\varepsilon} \varphi(u) \rightarrow H \varphi(u), \quad \varepsilon \rightarrow 0 .
$$

The limit exponential generator is given by

$$
H \varphi(u)=\frac{1}{2} \sigma^{2}\left[\varphi^{\prime}(u)\right]^{2}+\widehat{C}(u) \varphi^{\prime}(u) .
$$

The final three steps in the solution of the large deviations problem are done in the same way as that described in [5] or [4].

\section{BIBLIOGRAPHY}

1. V. S. Koroliuk and N. Limnios, Stochastic Systems in Merging Phase Space, World Scientific Publishing Co. Pte. Ltd., Hackensack, NJ, 2005. MR2205562 (2007a:60004)

2. V. S. Korolyuk and V. V. Korolyuk, Stochastic Models of Systems, Kluwer Academic Publishers, Dordrecht, 1999. MR.1753470 (2002b:60169)

3. S. N. Ethier and T. G. Kurtz, Markov Processes. Characterization and Convergence, John Wiley \& Sons, Inc., New York, 1986. MR838085 (88a:60130)

4. J. Feng and T. G. Kurtz, Large Deviations for Stochastic Processes, American Mathematical Society, Providence, RI, 2006. MR2260560 (2009g:60034)

5. M. J. Freidlin and A. M. Wentzel, Random Perturbation of Dynamical Systems, Springer Verlag, Berlin, 1998. MR 1652127 (99h:60128)

6. W. Feller, An Introduction to Probability Theory and Its Applications, Vol. II, John Wiley \& Sons Inc., New York-London-Sydney, 1966. MR.0210154 (35:1048)

7. I. I. Gikhman and A. V. Skorokhod, Introduction to the Theory of Random Processes, "Nauka", Moscow, 1977; English transl., Dover Publications, Inc., Mineola, NY, 1996. MR.1435501 $(97 \mathrm{j}: 60001)$

Institute of Mathematics, National Academy of Science of Ukraine, Tereshchenkivs'ka Street, 3, Kyiv 01601, Ukraine

E-mail address: korol@imath.kiev.ua

Received 10/MAY/2011

Translated by N. SEMENOV 